\title{
A new control method for Integral Processes with Inverse Response and Dead Time based on Disturbance Observer
}

\author{
Miao Wang ${ }^{1, a}$, Weidong Zhang ${ }^{1, b}$ \\ ${ }^{1}$ Key Laboratory of System Control and Information Processing, Ministry of Education of China, and \\ Department of Automation, Shanghai Jiao Tong University, Shanghai 200240, P.R. China \\ awangmiao46@sjtu.edu.cn, bwdzhang@sjtu.edu.cn
}

Keywords: Integral process, input load disturbance rejection, disturbance observer, $\mathrm{H}_{2}$ norm

\begin{abstract}
A new control method based on disturbance observer (DOB) is proposed to control the integral processes with inverse response and dead time. The main contribution of this paper is that the optimal analytical result in terms of $\mathrm{H}_{2}$ for the set-point controller and the disturbance observer are provided. And only two parameters are to be tuned respectively to achieve the set-point tracking performance specification and input load disturbance rejection specification. Simulation examples illuminate the effectiveness of the proposed design procedure.
\end{abstract}

\section{Introduction}

The integrating processes are very common in industrial and chemical areas. When the integral process is upset by a step signal input, the output will increase at a fixed speed until the physical limit is reached [1]. The presence of dead time and inverse response makes the control more difficult and interesting [2]. The researches on the control of the integrating process have been very active [3][4].

As was originally proposed by Ohnishi [5], the DOB control scheme has been often used in disturbance rejection control [6][7]. The main advantage of DOB is that it adds inner disturbance observer loop to the nominal feedback controller and decouples the disturbance response from the set point response. Zhong et al. [8] applies the DOB to control integral processes with dead time. And many papers have been published based on this structure [9][10].

Though many design procedures based on DOB have been proposed, few articles solve the input disturbance rejection problem, which is to minimize the criterion min $\|W(s) G(s) S(s)\|_{2}$, where $W(s)$ is the weighting function and $S(s)$ is the closed-loop sensitivity function. Sun et al. [11]propose a simple and effective controller formula which presents an analytical solution to the input disturbance rejection problem for the non-minimum phase processes based on the DOB control scheme. But the plants with imaginary axis poles are excluded in [11].

In this paper, we adopt the controller design methods from [11] and the control scheme based on DOB to control the integral processes with inverse response and dead time. The main contribution of this paper is that the optimal analytical result in terms of $\mathrm{H}_{2}$ for the set-point controller and the disturbance observer are provided. The rest of this paper is organized as follows. In Section 2, the control scheme is shown first. Then we propose the set of controllers and filters that guarantee the internal stability of the control system. And the optimal solutions for both the controllers and the DOB are presented based on the respective performance criterion in terms of $\mathrm{H}_{2}$ norm. In Section 3, we present the analytical result based on Section 2 for the integral processes with inverse response and dead time. Simulation results are shown in Section 4. Conclusions are made in Section 5 finally.

\section{Control Scheme}

The control scheme based on disturbance observer is shown in Fig.1, where $G_{p}(s) e^{-\theta s}$ is the real plant. $G_{p}(s)$ is the delay free part of the plant and $e^{-\theta s}$ is the delay part. $G_{m}(s)$ is the nominal model of $G_{p}(s) ; C(s)$ is the set-point response controller to be decided. $r(s), d(s), y(s), n(s)$ and $c(s)$ are the 
reference input, input disturbance, system output, measurement noise and the command signal, respectively. The $Q(s)$ is the filter of DOB to be designed.

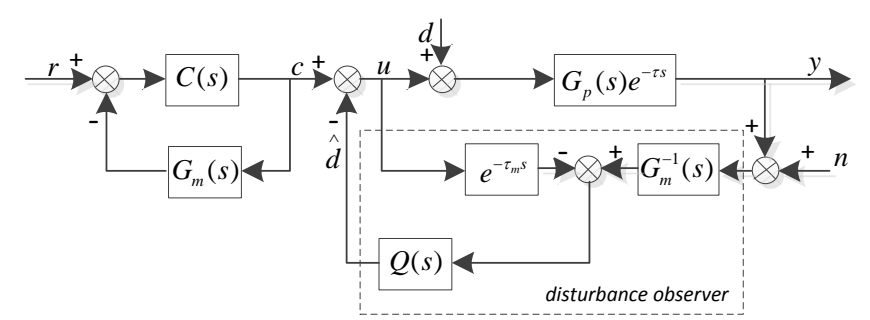

Fig.1: Control Scheme based on disturbance observer

Integrating plants have one or more poles at the origin. In this paper, it is assumed that they do not have open right half plane poles. Consider the integral process with inverse response and dead time:

$$
\begin{gathered}
G(s)=G_{p}(s) e^{-\theta s}=\frac{K N_{+}(s) N_{-}(s)}{s M_{-}(s)} e^{-\theta s} \\
N_{+}(s)=\prod_{i=1}^{n_{u}}\left(-\tau_{i} s+1\right)^{k_{i}} ; N_{-}(s)=\prod_{i=n_{u}+1}^{n_{v}}\left(\tau_{i} s+1\right)^{k_{i}} ; M_{-}(s)=\prod_{j=1}^{n_{w}}\left(\tau_{j} s+1\right)^{k_{j}} \quad \forall \tau_{i}, \tau_{j}>0
\end{gathered}
$$

Here $K$ is a real constant denoting the static gain, $\theta$ is a positive real constant denoting the time delay. The subscript "+" denotes the roots in the right half plane (RHP); and the subscript "-” denotes the roots in the left half plane (LHP). It is assumed that $N_{+}(0)=N_{-}(0)=M_{-}(0)=1$ and $\operatorname{deg}\left\{N_{+}(s)\right\}+$ $\operatorname{deg}\left\{N_{-}(s)\right\} \leq \operatorname{deg}\left\{M_{-}(s)\right\}+1$. Here $\operatorname{deg}\{\cdot\}$ denotes the degree of a polynomial.

\subsection{System Parameterization}

For control system, the minimal requirement is internal stability. In the nominal case, $G_{p}(s)=$ $G_{m}(s)$, the following function is defined $P(s)=C(s) /\left(1+C(s) G_{m}(s)\right)$.

The closed-loop system in Fig. 1 is internally stable if and only if all the elements in the transfer matrix $\boldsymbol{H}(s)$ are stable:

$$
\begin{aligned}
& \boldsymbol{H}(s)=\left[\begin{array}{ccc}
G_{m}(s) P(s) e^{-\theta_{m} s} & G_{m}(s) e^{-\theta_{m} s}\left(1-Q(s) e^{-\theta_{m} s}\right) & -Q(s) e^{-\theta_{m} s} \\
P(s) & -Q(s) e^{-\theta_{m} s} & -G_{m}^{-1}(s) Q(s)
\end{array}\right] \\
& Y(s)=H(s) X(s) ; Y(s)=\left[\begin{array}{ll}
y(s) & u(s)
\end{array}\right]^{\mathrm{T}} ; X(s)=\left[\begin{array}{lll}
r(s) & d(s) & n(s)
\end{array}\right]^{\mathrm{T}}
\end{aligned}
$$

Theorem 1 The control scheme shown in Fig.1 is internally stable if and only if: 1) $P(s)$ is stable and $G_{m}(s) P(s)$ is stable; 2$) G_{m}(s) e^{-\theta_{m} s}\left(1-Q(s) e^{-\theta_{m} s}\right)$ is stable; 3$) Q(s)$ is stable and $-G_{m}^{-1}(s) Q(s)$ is stable.

The $C(s)$ and $Q(s)$ that satisfies this requirement can be parameterized

$$
C(s)=\frac{P(s)}{1-P(s) G_{m}(s)}, \quad P(s)=\frac{s P_{1}(s)}{K}
$$

There is no zero-pole cancellation in the right half plane in $C(s)$. And

$$
Q(s)=Q_{1}(s) N_{+}(s), \quad \lim _{s \rightarrow 0}\left[1-Q(s) e^{-\theta_{m} s}\right]=0
$$

$P_{1}(s)$ and $Q_{1}(s)$ are stable transfer functions that make $P(s)$ and $Q(s)$ proper and $P_{1}(s)=Q_{1}(s)=1$.

To obtain zero-steady errors for a unit step reference signal and a unit load disturbance:

$$
\begin{gathered}
\lim _{s \rightarrow 0}\left[1-G_{m}(s) P(s) e^{-\theta_{m} s}\right]=0, \quad P_{1}(s)=1+s P_{2}(s) \\
\lim _{s \rightarrow 0} G_{m}(s) e^{-\theta_{m} s}\left[1-Q(s) e^{-\theta_{m} s}\right]=0 \\
\dot{Q}(0)=\theta_{m}, \quad \dot{Q}_{1}(0)=\theta_{m}-\dot{N}_{+}(0), \quad Q_{1}(s)=1+s Q_{2}(s)
\end{gathered}
$$

\subsection{Optimal Controller Design}

The design of the $\mathrm{H}_{2}$ optimal controller for the plant is illuminated in this section. To obtain the optimal set-point tracking performance, the performance criterion is $\min \left\|W(s) S_{r, e}(s)\right\|_{2}$. 
$W(s)$ is the weighting function. For the step input, $r(s)=1 / s$, we can choose $W(s)=1 / s$. While $S_{r, e}(s)$ is the transfer function from the reference input $r(s)$ to the error $e(s) . S_{r, e}(s)=1-G_{m}(s) P(s) e^{-\theta s}$.

Introduce the symbol $L_{2}$ for the family of all the rational transfer function with no poles on the imaginary axis. Let $\mathrm{H}_{2}$ denote the subset of $L_{2}, \mathrm{H}_{2}^{\perp}$ the set of rational transfer functions analytic in Re $s \leq 0$. Every function $F(s)$ in $L_{2}$ can be uniquely expressed as

$$
F=F_{1}+F_{2}, \quad F_{1} \in H_{2}, \quad F_{2} \in H_{2}^{\perp}, \quad\left\|F_{1}+F_{2}\right\|_{2}^{2}=\left\|F_{1}\right\|_{2}^{2}+\left\|F_{2}\right\|_{2}^{2}
$$

Thus, the performance criterion is equal to

$$
\min \left\|W(s) S_{r, e}(s)\right\|_{2}^{2}=\left\|\frac{1}{s}\left\{1-G_{m}(s) P(s) e^{-\theta s}\right\}\right\|_{2}^{2}=\left\|\frac{1}{s}\left\{1-\frac{N_{+}(s) N_{-}(s)}{M_{-}(s)}\left[1+s P_{2}(s)\right] e^{-\theta s}\right\}\right\|_{2}^{2}
$$

Theorem 2 The control scheme is shown in Fig. 1. The controller to optimize the set-point tracking performance criterion in terms of $H_{2}$ norm is given as

$$
C_{\text {opt }}(s)=\frac{P_{\text {opt }}(s)}{1-P_{o p t}(s) G_{m}(s)}, \quad P_{o p t}(s)=\frac{s M_{-}(s)}{K N_{+}(-s) N_{-}(s)}
$$

Proof: The time delay is all-pass, and the transfer function $N_{+}(s) / N_{+}(-s)$ is also all-pass. In light of the definition of the 2-norm, an all-pass transfer function does not affect the value of the 2-norm.

$$
\min \left\|W(s) S_{r, e}(s)\right\|_{2}^{2}=\left\|\frac{e^{\theta s} N_{+}(-s)-N_{+}(s)}{s N_{+}(s)}+\frac{M_{-}(s)-N_{+}(-s) N_{-}(s)}{s M_{-}(s)}-\frac{N_{+}(-s) N_{-}(s) P_{2}(s)}{M_{-}(s)}\right\|_{2}^{2}
$$

Because $N_{+}(0)=N_{-}(0)=M_{-}(0)=1, s$ is a factor of $e^{\theta s} N_{+}(-s)-N_{+}(s)$ and $M_{-}(s)-N_{+}(-s) N_{-}(s)$. Therefore,

$$
\min \left\|W(s) S_{r, e}(s)\right\|_{2}^{2}=\left\|\frac{e^{\theta s} N_{+}(-s)-N_{+}(s)}{s N_{+}(s)}\right\|_{2}^{2}+\left\|\frac{M_{-}(s)-N_{+}(-s) N_{-}(s)\left[1+s P_{2}(s)\right]}{s M_{-}(s)}\right\|_{2}^{2}
$$

Minimizing the right-hand side, the optimal $P_{2 o p t}(s)$ is

$$
P_{2 o p t}(s)=\frac{M_{-}(s)-N_{+}(-s) N_{-}(s)}{s N_{+}(-s) N_{-}(s)}, \quad P_{\text {opt }}(s)=\frac{s M_{-}(s)}{K N_{+}(-s) N_{-}(s)}
$$

which is the optimal solution. Introduce the filter $J_{C}(s)$, then

$$
P(s)=P_{o p t}(s) J_{C}(s)=\frac{s M_{-}(s)}{K N_{+}(-s) N_{-}(s)} J_{C}(s)
$$

To achieve the zero-steady errors for a unit step reference,

$$
\lim _{s \rightarrow 0}\left[1-G_{m}(s) P(s) e^{-\theta_{m} s}\right]=0, \quad J_{C}(s)=\frac{1}{\left(\lambda_{C} s+1\right)^{n_{j}}}
$$

where $\lambda_{C}$ is called the performance degree, and $n_{j}$ should be large enough to make $P(s)$ proper.

\subsection{Optimal DOB Design}

To obtain the optimal input load disturbance rejection, the performance criterion is $\min \left\|W(s) S_{d, y}(s)\right\|_{2}$.

For the step input disturbance, $d(s)=1 / s$, we can choose $W(s)=1 / s$. And $S_{d, y}(s)$ is the transfer function from the input disturbance $d(s)$ to the output $y(s) . S_{d, y}(s)=G_{m}(s) e^{-\theta_{m} s}\left[1-Q(s) e^{-\theta_{m} s}\right]$.

Theorem 3 The control scheme is shown in Fig.1. The DOB to optimize the input load disturbance rejection criterion in terms of $\mathrm{H}_{2}$ norm is given as

$$
Q_{o p t}(s)=\frac{[s Y(s)+1] N_{+}(s)}{K N_{+}^{2}(-s) N_{-}(s)}
$$

where $Y(s)$ is a rational polynomial and satisfies the following conditions: 1) $Y(s)$ doesn't contain prediction; 2) The order of $Y(s)$ is not more than $\operatorname{deg}\left\{M_{-}(s)\right\}+1$.

Proof: 


$$
\begin{aligned}
& \left.\min \left\|W(s) S_{d, y}(s)\right\|_{2}^{2}=\| W(s) G_{m}(s) e^{-\theta_{m} s}\left[1-Q(s) e^{-\theta_{m} s}\right]\right\} \|_{2}^{2} \\
& =\left\|\frac{K N_{+}^{2}(-s) N_{-}(s) e^{\theta_{m} s}-N_{+}(s)-s X(s)}{s^{2} N_{+}(s) M_{-}(s)}+\frac{X(s)}{s N_{+}(s) M_{-}(s)}+\frac{1-K N_{+}^{2}(-s) N_{-}(s)-K s N_{+}^{2}(-s) N_{-}(s) Q_{2}(s) \|^{2}}{s^{2} M_{-}(s)}\right\|_{2}
\end{aligned}
$$

$X(s)$ should satisfy the conditions: 1$) s^{2} M_{-}(s)$ is a factor of $\left.K N_{+}^{2}(-s) N_{-}(s) e^{\theta_{m} s}-N_{+}(s)-s X(s) ; 2\right)$ $N_{+}(s)$ is a factor of $X(s)$. If $X(s)$ states as $X(s)=N_{+}(s) Y(s)$, the criterion can be expressed as

$$
\left\|\frac{K N_{+}^{2}(-s) N_{-}(s) e^{\theta_{m} s}-N_{+}(s)-s X(s)}{s^{2} N_{+}(s) M_{-}(s)}\right\|_{2}^{2}+\left\|\frac{X(s)}{s N_{+}(s) M_{-}(s)}+\frac{1-K N_{+}^{2}(-s) N_{-}(s)-K s N_{+}^{2}(-s) N_{-}(s) Q_{2}(s)}{s^{2} M_{-}(s)}\right\|_{2}^{2}
$$

Minimizing the right-hand side, we have

$$
Q_{2}(s)=\frac{s Y(s)+1-K N_{+}^{2}(-s) N_{-}(s)}{K s N_{+}^{2}(-s) N_{-}(s)}
$$

which leads to

$$
Q_{1}(s)=\frac{s Y(s)+1}{K N_{+}^{2}(-s) N_{-}(s)}, \quad Q_{o p t}(s)=\frac{[s Y(s)+1] N_{+}(s)}{K N_{+}^{2}(-s) N_{-}(s)}
$$

\section{Application for the Integral Processes with Inverse Response and Dead Time}

To obtain $Q_{o p t}(s)$, the polynomial $Y(s)$ is the only element to be decided. To simplify the result, we adopt the following process as an example. And the result is easy to be expanded into more difficult situations. The integral process with inverse response and dead time is expressed as

$$
G(s)=\frac{K N_{+}(s)}{s M_{-}(s)} e^{-\theta s}=\frac{K(-\alpha s+1)}{s(\tau s+1)} e^{-\theta s}
$$

Theorem 4 The control scheme is shown in Fig.1. If the plant is expressed as (23), the DOB to optimize the input load disturbance rejection criterion in terms of $H_{2}$ norm is given as

$$
\begin{gathered}
Q_{o p t}(s)=\frac{[s Y(s)+1] N_{+}(s)}{K N_{+}^{2}(-s)}=\frac{[s Y(s)+1](-\alpha s+1)}{K(\alpha s+1)^{2}} \\
Y(s)=\frac{K \tau\left[\theta+3 \alpha-\tau+\frac{(-\alpha+\tau)^{2} e^{-\theta / \tau}}{\alpha+\tau}\right] s^{2}+(K \theta+3 K \alpha) s+K-1}{s}
\end{gathered}
$$

\section{Proof:}

To satisfy the first conditions that $X(s)$ possesses, equivalently, it leads to

$$
\begin{gathered}
\lim _{s \rightarrow-1 / \tau}\left[K N_{+}^{2}(-s) e^{\theta_{m} s}-N_{+}(s)-s X(s)\right]=0 \\
\lim _{s \rightarrow 0}\left[K N_{+}^{2}(-s) e^{\theta_{m} s}-N_{+}(s)-s X(s)\right]=0 \\
\lim _{s \rightarrow 0} \frac{d}{d s}\left[K N_{+}^{2}(-s) e^{\theta_{m} s}-N_{+}(s)-s X(s)\right]=0
\end{gathered}
$$

According to (26) and (27),

$$
Y(s)=\frac{1}{s}\left\{K\left[1-\frac{(-\alpha / \tau+1)^{2} e^{-\theta / \tau}}{\alpha / \tau+1}\right] \cdot \tau s+K-1+B(s)\right\}
$$

where $B(s)$ satisfies equation (28) and $\lim _{s \rightarrow 0,-1 / \tau} B(s)=0$. Therefore,

$$
B(s)=K \tau\left[\theta+3 \alpha-\tau+\frac{(-\alpha+\tau)^{2} e^{-\theta / \tau}}{\alpha+\tau}\right] s^{2}+(K \theta+3 K \alpha-a \tau) s
$$

By submitting (30) into (29), it leads to the equation (25). Proved.

Similar to the design for the $C(s)$, a filter $J_{q}(s)$ is introduced to $Q_{o p t}(s)$ and should make $Q(s)$ and $-G_{m}^{-1}(s) Q(s)$ proper. Therefore on this condition, the relative degree of $J_{q}(s)$ should be no less than 2 . 
To achieve the zero-steady errors for the step input load disturbance, the filter $J_{q}(s)$ is:

$$
J_{q}(s)=\frac{3 \lambda_{q} s+1}{\left(\lambda_{q} s+1\right)^{3}}
$$

\section{Examples}

Consider the following process[12]:

$$
\begin{gathered}
G(s)=0.547 \frac{-0.418 s+1}{s(1.06 s+1)} e^{-0.1 s} \\
C(s)=\frac{1.06 s+1}{0.547\left(0.418 \lambda_{c} s+\lambda_{c}+2 * 0.418\right)} \\
Q(s)=\frac{\left[0.5806 s^{2}+1.3540 s+1\right](-0.418 s+1)\left(3 \lambda_{q} s+1\right)}{(0.418 s+1)^{2}\left(\lambda_{q} s+1\right)^{3}}
\end{gathered}
$$

The first simulation demonstrates the controller $C(s)$ for the set-point tracking ability. The reference set-point input is a step signal, $r(s)=1 / s$ at $t=1(s)$. The process output and the controller output of the nominal case are shown in Figs.(2-3), where the performance degree $\lambda_{c}$ equals to 2, 3, 4, respectively. $\lambda_{q}$ is set sufficiently large as $\lambda_{q}=4$ while the input disturbance $d(s)=0$.

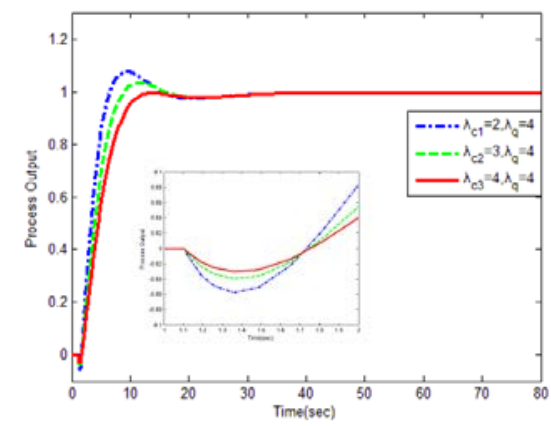

Fig.2: Process output of nominal set-point response

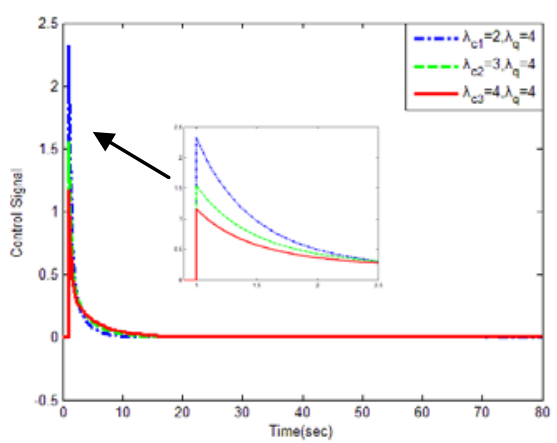

Fig.3: Control output of nominal set-point response

The simulation results shows that with the increase of $\lambda_{c}$, the process output provides smaller inverse overshoot but longer settling time and the controller costs less energy, which contributes to better robustness for the system.

The second simulation tests the input load disturbance rejection ability of the DOB-based control scheme. The reference input is the same as in the first simulation, $r(s)=1 / s$ at $t=1(s)$ and the input load disturbance is chosen as $d(s)=0.1 / s$ at $t=30(s)$. The parameter $\lambda_{q}$ of $Q(s)$ is set as 3 , 4, and 5 with settled $\lambda_{c}=3$.

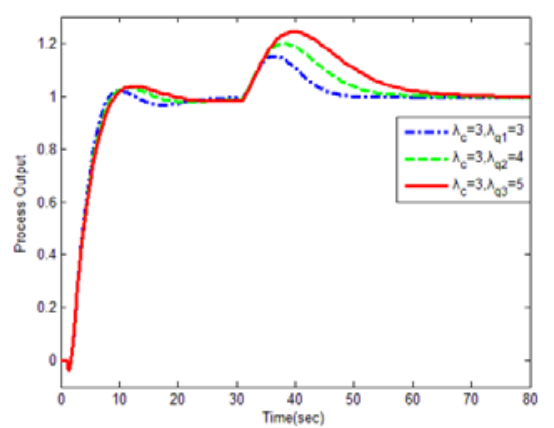

Fig.4: Process output of input load disturbance response

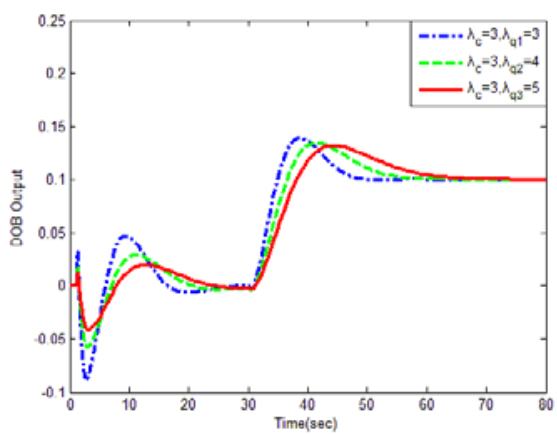

Fig.5: DOB output of input load disturbance response

Figs.(4-5) show the process output and the DOB output with the input load disturbance injected into the system. The simulation results testify that the system achieves good set-point tracking 
response and input load disturbance rejection abilities in the DOB-based control scheme. A smaller $\lambda_{q}$ provides process output with quicker response and smaller peak value for disturbance response, but it costs more control efforts and severe response for DOB output.

\section{Conclusions}

In this paper, the control scheme based on disturbance observer is adopted for the integral processes with inverse response and dead time. The main contribution of this paper is that the optimal analytical result of the input disturbance rejection criterion for the integral processes with inverse response and dead time has been derived. Optimal analytical solutions in terms of $\mathrm{H}_{2}$ for the set-point controller and the disturbance observer are provided. The simulation examples illuminate the effectiveness of the proposed design procedure. In fact it is a two degree of freedom control structure and only two parameters are to be tuned respectively to achieve the set-point tracking performance specification and input load disturbance rejection specification.

\section{Acknowledgements}

This paper is partly supported by the National Science Foundation of China (61025016, 61473183, 61034008, 61221003), Program of Shanghai Subject Chief Scientist (14XD1402400), and SJTU M\&E Joint Research Foundation (YG2013MS04).

\section{References}

[1] Zhang W. Quantitative Process Control Theory[M]. Boca Raton: CRC Press, 2012.

[2] Luyben W L. Identification and tuning of integrating processes with dead-time and inverse response[J]. Industrial \& engineering chemistry research, 2003, 42(13): 3030-3035.

[3] Normey-Rico J E, Camacho E F. Robust tuning of dead-time compensators for processes with an integrator and long dead-time[J]. Automatic Control, IEEE Transactions on, 1999, 44(8): 1597-1603.

[4] Åström K J, Hang C C, Lim B C. A new Smith predictor for controlling a process with an integrator and long dead-time[J]. IEEE transactions on Automatic Control, 1994, 39(2): 343-345.

[5] Ohnishi K, et al. Microprocessor-controlled DC motor for load-insensitive position servo system[J]. Industrial Electronics, IEEE Transactions on, 1987 (1): 44-49..

[6] Sariyildiz E, Ohnishi K. Performance and robustness trade-off in disturbance observer design[C]//Industrial Electronics Society, IECON 2013-39th Annual Conference of the IEEE. IEEE, 2013: 3681-3686.

[7] Choi Y, Yang K, Chung W K, et al. On the robustness and performance of disturbance observers for second-order systems[J]. IEEE Transactions on Automatic Control, 2003, 48(2): 315-320.

[8] Zhong Q C, Normey-Rico J E. Control of integral processes with dead-time. Part 1: Disturbance observer-based 2DOF control scheme[J]. IEE Proceedings-Control Theory and Applications, 2002, 149(4): 285-290.

[9] Hongdong Z, Guanghui Z, Huihe S. Control of the process with inverse response and dead-time based on disturbance observer[C]//American Control Conference, 2005. Proceedings of the 2005. IEEE, 2005: 4826-4831.

[10]Kadam S D, Waghmare L M. Control of integrating processes with dead-time using PID controllers with disturbance observer based smith predictor[C]//Control Applications (CCA), 2013 IEEE International Conference on. IEEE, 2013: 1265-1269.

[11] Sun B, Zhang W, Zhang W, et al. Input load disturbance rejection of non-minimum phase processes using Disturbance Observer Control Scheme[C]//Control Conference (CCC), 2013 32nd Chinese. IEEE, 2013: 144-149.

[12]Luyben W L. Identification and tuning of integrating processes with deadtime and inverse response[J]. Industrial \& engineering chemistry research, 2003, 42(13): 3030-3035. 